\title{
Symptomatic congenital syphilis in a tertiary neonatal unit in Cape Town, South Africa: High morbidity and mortality in a preventable disease
}

\author{
S Pillay, MB BCh, FC Paed (SA), Cert Neonatology (SA), MMed (Paeds), DCH; \\ L J Tooke, MB ChB, FC Paed (SA), Cert Neonatology (SA), MMed (Paeds) \\ Department of Neonatology, Groote Schuur Hospital and Faculty of Health Sciences, University of Cape Town, South Africa
}

Corresponding author: S Pillay (shakti.pill@yahoo.com)

\begin{abstract}
Background. Despite preventive measures and effective treatment, congenital syphilis continues to impact significantly on neonatal morbidity and mortality. There has been no recent South African (SA) published literature reviewing congenital syphilis, particularly in the context of a tertiary neonatal setting.

Objectives. To describe the clinical features of symptomatic neonates with congenital syphilis and to identify modifiable patient, clinical and health facility factors that contributed to syphilis infection.

Methods. All positive serological tests for syphilis performed in neonates at Groote Schuur Hospital (GSH), Cape Town, SA, between 1 January 2011 and 31 December 2013 were obtained. Folders were reviewed, and neonates with clinical signs of congenital syphilis were included. Results. Of 50 symptomatic neonates, 19 (38\%) died. Twenty-eight mothers (56\%) were unbooked and therefore received no antenatal care. Most mothers (98\%) were inadequately treated. Health worker-related failures included poor notification and partner tracing as well as failure to recheck syphilis serology after 32 weeks' gestation in mothers who initially tested negative. Thirty-four neonates required intensive care unit admission. Two significant predictors of mortality were 1-minute and 5-minute Apgar scores $<5$. Hydrops fetalis was an independent risk factor for mortality, as were moderate to severely abnormal cranial ultrasound scan findings.

Conclusions. Congenital syphilis in neonates admitted to the GSH neonatal unit was associated with substantial morbidity and mortality. The modifiable factors identified represent inadequate antenatal healthcare and health system failures. These factors are longstanding, highlighting the need to establish governance and audit processes and address the continuing socioeconomic and sociocultural barriers that mothers face as a way forward in ultimately eliminating this entirely preventable disease.
\end{abstract}

S Afr Med J 2019;109(9):652-658. https://doi.org/10.7196/SAMJ.2019.v109i9.13817

Mother-to-child transmission of syphilis is associated with both adverse pregnancy outcomes (fetal loss, stillbirth, neonatal death, preterm birth and low birthweight) and congenital syphilis. ${ }^{[1]}$ In 2012, an estimated 930000 maternal syphilis infections caused 350000 adverse pregnancy outcomes globally. ${ }^{[2]}$ The greatest burden of syphilis in pregnancy occurred in Africa. ${ }^{[2]}$ Despite the availability of affordable preventive tools for over 50 years, the global perinatal mortality rate from congenital syphilis exceeds the perinatal deaths estimated for HIV and tetanus. ${ }^{[3,4]}$ Globally, in 2016, 38000 neonates died from congenital syphilis. ${ }^{[5]}$ The World Health Organization Strategy for the Global Elimination of Congenital Syphilis was launched in 2007, calling for political commitment in addressing syphilis screening, treatment and surveillance. However, in the past 12 years, little progress has been made in eliminating this preventable disease. ${ }^{[6]}$

The prevalence of syphilis in South Africa (SA) is not declining, with the prevalence rate among women presenting for antenatal care having risen from $1.6 \%$ in 2011 to $2.0 \%$ in $2015 .{ }^{[7]}$ Data from the Perinatal Problem Identification Program parallel these figures, showing a steady increase in the percentage of births in serologically positive pregnant mothers in the Metro West area of Western Cape Province, with $0.8 \%$ of births occurring in serologically positive mothers in 2011, $0.9 \%$ in 2012 and $1.2 \%$ in 2013. In addition, perinatal deaths from congenital syphilis in the Metro West area during the same time period showed an increase from $3 \%$ to $3.5 \% .{ }^{[8]}$ In May 2017, nearly 40 years after the initiation of a national syphilis screening programme, the South African National AIDS Council released the fourth National Strategic Plan, one of the aims of which is to eliminate congenital syphilis. ${ }^{[9]}$

There has been no recent SA published literature reviewing congenital syphilis, particularly in the context of a tertiary neonatal setting. However, in 1996 the Baragwanath Hospital (Johannesburg) experience of 58 cases of congenital syphilis admitted to an intensive care unit (ICU) was published in conference proceedings. The mortality rate was $29 \%$ and all 6 infants with hydrops fetalis died. There was substantial morbidity, in particular intraventricular haemorrhage (IVH), and need for vasopressors. ${ }^{[10]}$ Given the paucity of recent data in SA, we performed a retrospective review of symptomatic congenital syphilis cases admitted to a tertiary hospital in Cape Town.

\section{Objectives}

To: (i) describe the clinical and laboratory features of symptomatic neonates with congenital syphilis, including the maximum level of care, interventions required and outcomes in terms of morbidity and mortality; and (ii) identify modifiable patient, personnel and health facility factors contributing to congenital syphilis infection. Patient factors (maternal) were defined as unbooked mothers and mothers who defaulted follow-up, while personnel and health facility factors were health worker failure to act on positive results or failure to test for syphilis. 


\section{Methods \\ Setting}

A retrospective review of neonates with symptomatic congenital syphilis admitted to the Groote Schuur Hospital (GSH) neonatal unit from 1 January 2011 to 31 December 2013 was performed. This neonatal unit has $\sim 2000$ admissions per annum and acts as the tertiary referral centre for the Metro West area of Cape Town, with a total of $\sim 40000$ deliveries per annum. ${ }^{[11]}$ The GSH treatment protocol for syphilis-exposed neonates is dependent on maternal treatment status and neonatal symptoms. A mother is considered fully treated once she has received three weekly intramuscular doses of benzathine penicillin, with the third dose $>1$ month before delivery. Asymptomatic neonates born to untreated or partially treated mothers receive a single intramuscular dose of benzathine penicillin. Neonates with signs of congenital syphilis, regardless of maternal treatment status, undergo serological testing and receive a full 10-day course of intravenous penicillin G.

\section{Data collection and analysis}

All positive syphilis serological tests performed at GSH in the neonatal population between 1 January 2011 and 31 December 2013 were obtained from the National Health Laboratory Service. Tests included rapid plasma reagin (RPR) and Treponema pallidum haemagglutination assay. Data from these neonatal folders were captured onto a standardised collection sheet. Potential modifiable factors were identified. Our study included neonates who tested serologically positive for syphilis (positive RPR with a titre $>1: 1$ ) with clinical signs of congenital syphilis.

All data were analysed descriptively, including means and standard deviations (SDs) for normally distributed data and medians and interquartile ranges (IQRs) for non-normally distributed continuous data. Descriptive statistics and graphical representation were carried out using StatPlus:mac (AnalystSoft, USA, 2016). Relative risks with 95\% confidence intervals (CIs) and $p$-values were used to compare outcomes between deaths and survivors. Bivariate analysis was used to compare differences in the death and survivor groups: $\chi^{2}$ tests for categorical data, $t$-tests for normally distributed numerical data, and the Mann-Whitney $U$-test for non-parametric numerical data. Shapiro-Wilk testing was used to assess whether data were normally distributed.

\section{Ethical considerations}

Ethical approval was obtained prior to commencement of the study from the University of Cape Town Faculty of Health Sciences Human Research Ethics Committee (ref. no. 263/2014).

\section{Results}

Eighty neonates tested serologically positive for syphilis. Of these, 50 were symptomatic and were included in the study (Fig. 1). Nineteen (38\%) of the symptomatic neonates died.

\section{Maternal characteristics}

Table 1 summarises the maternal characteristics. The mean (SD) maternal age was 24.8 (5.5) years. Most mothers (60\%) were multiparous. Twenty-eight (56\%) were unbooked (received no antenatal care (ANC)). The mean (SD) age of the unbooked mothers was 24.8 (4.5) years; 10 were primiparous and 11 used substances. Twenty-two mothers (44\%) did book, but the median gestation at booking was 30 weeks (IQR 23.5 - 31.5). Overall, the maternal treatment rate was poor. Thirty-nine mothers $(78 \%)$ were untreated; 28 sought no antenatal care and were therefore undiagnosed until

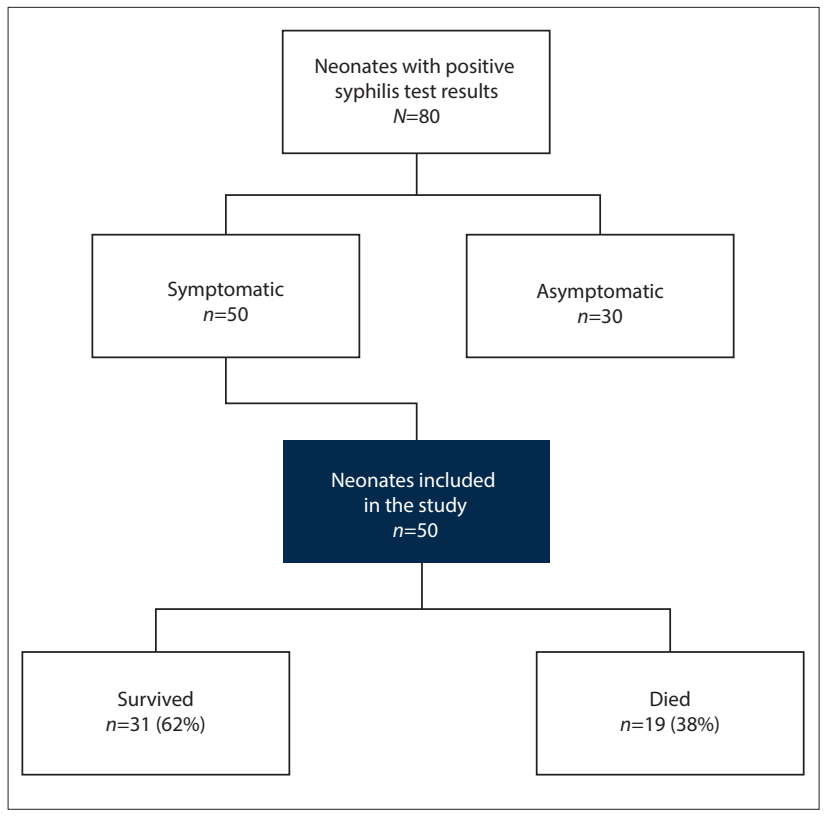

Fig. 1. Summary of the study results.

delivery, and 11 infected mothers who initially tested negative were undiagnosed owing to failure of re-testing after 32 weeks' gestation as per the National Department of Health (NDoH) protocol. This protocol is not being implemented routinely in the Western Cape. Ten mothers were partially treated, with 9 initiating but not completing penicillin treatment owing to preterm delivery and 1 failing to return for follow-up. One mother, who sought antenatal care at 22 weeks' gestation, completed treatment. As her partner was neither traced nor treated, maternal reinfection was likely. Overall partner tracing was poor, with 4 partners (8\%) documented traced and treated.

\section{Neonatal characteristics}

Gestational age and birth weight

Table 2 summarises the neonatal characteristics. The median gestational age was 34 weeks based on modified Ballard scoring ${ }^{[12]}$ (IQR 31 - 36.5), with a mean (SD) birth weight of 1933 (685) g. There were no statistically significant differences between infants who died and those who survived in terms of gestational age $(p=0.15)$ or birth weight ( $p=0.08)$. Twenty-seven neonates $(54 \%)$ were notified.

\section{Resuscitation at birth}

Twenty-five neonates (50\%), including all 19 neonates who died, required resuscitation at birth. The median 1- and 5-minute Apgar scores of those who died were 3 (IQR 2 - 5) and 5 (IQR $3-6$ ), respectively, compared with 5 (IQR 2 - 6) and 7 (IQR 5 - 8) in the cohort as a whole. Neonates with a low 1-minute Apgar score of $<5$ were 3.5 times more likely to die than neonates with a 1 -minute Apgar score of $\geq 5$ (relative risk (RR) 3.5, 95\% CI 1.6 - 7.7). Following resuscitation, a 5-minute Apgar score that remained $<6$ was associated with an increased risk of mortality (Table 3). Blood gases obtained from the umbilical cord at delivery were known for 11 of the neonates who died; the mean (SD) pH was $7.0(0.16)$.

\section{Clinical signs and special investigations}

The three commonest clinical signs were respiratory distress, hepatosplenomegaly (HSM) and petechiae (Fig. 2). A total of 11 neonates were hydropic, of whom 8 (73\%) died. Hydropic neonates 


\begin{tabular}{|c|c|c|c|}
\hline Variable & Total $(N=50), n(\%)$ & Booked $(N=22), n(\%)$ & Unbooked $(N=28), n(\%)$ \\
\hline \multicolumn{4}{|l|}{ Age (years) } \\
\hline$\leq 20$ & $16(32)$ & $9(41)$ & $7(25)$ \\
\hline $21-25$ & $11(22)$ & $3(14)$ & $8(29)$ \\
\hline $26-30$ & $16(32)$ & $6(27)$ & $10(36)$ \\
\hline $31-35$ & $5(10)$ & $2(9)$ & $3(11)$ \\
\hline $36-40$ & $2(4)$ & $2(9)$ & 0 \\
\hline \multicolumn{4}{|l|}{ Substance use } \\
\hline Yes & $12(24)$ & $1(5)$ & $11(39)$ \\
\hline No & $38(76)$ & $21(95)$ & $17(61)$ \\
\hline \multicolumn{4}{|l|}{ Gravidity } \\
\hline 1 & 20 & $10(45)$ & $10(36)$ \\
\hline 2 & 13 & $6(27)$ & $7(25)$ \\
\hline 3 & 8 & $2(9)$ & $6(21)$ \\
\hline 4 & 4 & $1(5)$ & $3(11)$ \\
\hline 5 & 2 & $2(9)$ & 0 \\
\hline 6 & 2 & $1(5)$ & $1(4)$ \\
\hline 7 & 1 & 0 & $1(4)$ \\
\hline \multicolumn{4}{|l|}{ HIV-positive } \\
\hline Yes & $7(14)$ & $3(14)$ & $4(14)$ \\
\hline No & $43(86)$ & $19(86)$ & $24(86)$ \\
\hline \multicolumn{4}{|c|}{ Antenatal care received } \\
\hline Yes & & 22 & 0 \\
\hline No & & 0 & 28 \\
\hline \multicolumn{4}{|c|}{ Gestational age at booking in booked mothers (weeks) } \\
\hline$<12$ & & $1(5)$ & \\
\hline $13-22$ & & $2(9)$ & \\
\hline $23-30$ & & $6(27)$ & \\
\hline $31-35$ & & $5(23)$ & \\
\hline$>36$ & & $2(9)$ & \\
\hline Unknown & & $6(27)$ & \\
\hline \multicolumn{4}{|c|}{ Treatment completed } \\
\hline Yes & $1(2)$ & $1(5)$ & 0 \\
\hline No & $49(98)$ & $21(95)$ & $28(100)$ \\
\hline \multicolumn{4}{|c|}{ Partner tracing } \\
\hline Yes & $4(8)$ & $1(5)$ & $3(11)$ \\
\hline No & $46(92)$ & $21(95)$ & $25(89)$ \\
\hline
\end{tabular}

(fluid collection in two or more sites) had an increased risk of mortality (RR 2.6, 95\% CI 1.4 - 4.8).

Long-bone X-rays were performed in 33 neonates (66\%), of whom 18 (55\%) had long-bone changes suggestive of congenital syphilis. These included metaphyseal translucent bands, periosteal reactions and the 'rat-bitten' appearance of Wimberger's sign. The bones most commonly affected were the tibia, femur and humerus.

Twenty-one neonates $(42 \%)$ were anaemic (haemoglobin $(\mathrm{Hb})$ $<11 \mathrm{~g} / \mathrm{dL}$ ) (median birth Hb $9.5 \mathrm{~g} / \mathrm{dL}$ (IQR 1.8 - 10.5), compared with a median birth $\mathrm{Hb} 12 \mathrm{~g} / \mathrm{dL}$ (IQR 10 - 15) for the entire cohort). Twenty neonates $(40 \%)$ were thrombocytopenic (platelet count $<150 \times 10^{9} / \mathrm{L}$ ) (median birth platelet count $27 \times 10^{9} / \mathrm{L}$ (IQR $16-37.5$ ), compared with a median birth platelet count of $140 \times 10^{9} / \mathrm{L}$ (IQR $30-193$ ) for the entire cohort). Forty of the 49 neonates in whom C-reactive protein (CRP) testing was performed within 48 hours of birth had CRP values $>10 \mathrm{mg} / \mathrm{L}$ (median CRP $63 \mathrm{mg} / \mathrm{L}$, IQR 38 - 107). This was unlikely to have been attributable to sepsis, as none had early positive blood cultures. Hypoglycaemia (blood glucose $<2.6 \mathrm{mmol} / \mathrm{L}$ ) was the commonest laboratory derangement, occurring in 27 neonates
(54\%). Fifteen neonates had severe hypoglycaemia (blood glucose $<2 \mathrm{mmol} / \mathrm{L}$ ): 4 were managed with glucagon or hydrocortisone, and 11 required central infusions of higher dextrose-containing solutions.

Thirty-two neonates had a cranial ultrasound scan (CUS) performed by senior clinicians, of which 23 (72\%) were abnormal in varying degrees (Table 3). Periventricular flares and grade 1 and 2 IVHs (Papile grading ${ }^{[13]}$ ) were considered insignificant, with no neurological sequelae on discharge in all surviving neonates. Coagulopathy at birth requiring blood products (bleeding with abnormal coagulation profile) was associated with a statistically significant increased risk of mortality (RR 2.2, 95\% CI $1.1-4.3$ ), as were moderate to severely abnormal CUS findings (RR 3.5, 95\% CI 1.8 - 6.9) (Table 4).

\section{ICU management}

Thirty-four neonates (68\%) were admitted to the ICU, of whom 29 (85\%) were intubated and 19 (56\%) required inotropic support. Premature neonates were not significantly more likely to be intubated than term neonates (RR 1.2, 95\% CI $0.5-2.5$ ). The need 


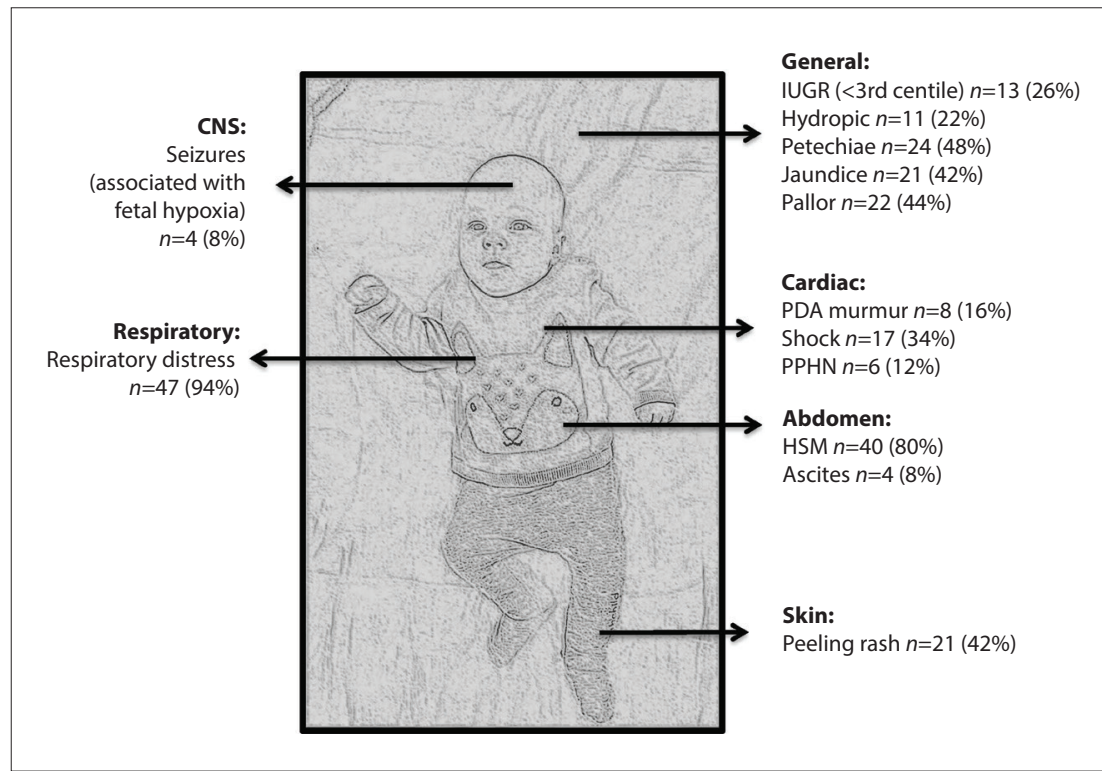

Fig. 2. Clinical signs in the 50 symptomatic neonates. (IUGR = intrauterine growth restriction; $P D A=$ patent ductus arteriosus; $P P H N=$ persistent pulmonary hypertension of the newborn; HSM = hepatosplenomegaly; CNS = central nervous system.)

for intubation within 24 hours of birth was associated with an increased risk of mortality $(p=0.009)$, as was the need for inotropes $(p=0.006)$.

\section{Discussion}

Over a 3-year period, 50 symptomatic neonates with congenital syphilis were admitted to the GSH neonatal unit, with $68 \%$ requiring intensive care. Despite maximal intervention, the mortality rate was high at $38 \%$.

The study highlighted numerous modifiable elements, the most notable relating to the maternal treatment rate. Congenital syphilis as a consequence of untreated mothers has been noted in case series from both high- and low-income countries. ${ }^{[14-16]}$ However, in our study population it was failure to access antenatal care, as evidenced by the high proportion of mothers $(56 \%)$ who were unbooked, with those who did book doing so close to delivery, that accounted for the inadequate treatment rate. Poor maternal health-seeking behaviour has been demonstrated in other developing countries. ${ }^{[14,15,17]}$ A recent SA study from Eastern Cape Province found that late ANC bookings were influenced by healthcare system-related issues, socioeconomic factors, failure of family planning services, and women's perceptions and lack of knowledge outweighing the perceived benefit of early ANC visits. ${ }^{[18]}$ In our study, there was poor documentation for why mothers were unbooked. All the mothers lived in urban areas near health facilities, and healthcare for pregnant women is free; however, access to healthcare in our setting is influenced by many factors, which include long waiting times, overburdened clinics and inability to negotiate time away from work. Lack of education and insight may have contributed, as the unbooked mothers tended to be of younger age with a third primiparous, findings supported by other studies. ${ }^{[19,20]}$ Substance use was a possible contributor, as most mothers who used substances were unbooked. Considering that multiparous women with term deliveries were also unbooked, there may have been underlying unidentified sociocultural factors at play. The influence of social factors on maternal antenatal care has been noted in studies from both the developing and developed world, with incarceration, substance use and prostitution mentioned as possible contributors. $^{[21,22]}$ However, social factors may be more complex and multifaceted; for example, Almeida et al. ${ }^{[17]}$ identified married women with a single sexual partner and $>8$ years of education as having high rates of syphilis.

Health worker-related failures to adhere to $\mathrm{NDoH}$ protocol and repeat syphilis testing after 32 weeks' gestation in mothers who booked early and tested negative at booking also resulted in untreated mothers $(22 \%)$. An initial negative test may have been a true negative, with maternal infection occurring later in pregnancy, or a false negative due to testing before seroconversion or test failure. Rapid tests, which are widely used in primary care settings, only have a sensitivity of $93-98 \% .^{[23]}$ Our study highlighted deficiency in partner tracing and treatment, a similar finding to those in two Brazilian studies. ${ }^{[17,24]}$ Herein lie important public health initiatives to address in order to attain the World Health Organization target for syphilis elimination of $>95 \%$ coverage of syphilis testing for pregnant women. ${ }^{[25]}$ More importantly, maternal syphilis is an independent risk factor for mortality in neonates and infants. ${ }^{[26]}$

The study findings of HSM, petechiae, pallor and jaundice were common clinical signs in symptomatic groups of neonates in other study populations. ${ }^{[14,21,27]}$ However, none of our patients had rhinitis or the 'syphilitic snuffles', which is quoted to affect $4-22 \%$ of neonates and may be the earliest presenting sign. ${ }^{[14,28]}$ Radiological abnormalities, despite being noted in more than half of the neonates who had long-bone $\mathrm{X}$-rays performed, were lower than rates of $75-100 \%$ quoted in the literature. ${ }^{[28,29]}$ Respiratory distress was the commonest clinical sign in this study. Considering the largely unbooked maternal population, it is likely that premature neonates were steroid immature. Differentiating between hyaline membrane disease (HMD) and pneumonia secondary to syphilis poses diagnostic difficulties, and they are often indistinguishable on chest X-rays. ${ }^{[30]}$ The preterm neonates treated as cases of HMD may have had syphilitic pneumonia, or vice versa.

Hypoglycaemia was one of the commonest laboratory derangements (54\%), noted in more than half of this study population. The period of greatest risk was within the first 24 hours after birth. Hypoglycaemia may be related to increased metabolic demands, reduction in glucose stores and/ or hepatic dysfunction. There have been case reports of hypopituitarism as a result of early congenital syphilis, which may cause prolonged hypoglycaemia in these neonates; however, this was unlikely in our study population owing to resolution of hypoglycaemia with no systemic features suggestive of hypopituitarism. ${ }^{[31]}$ These findings indicate that despite hypoglycaemia not being a statistically significant risk factor for death, it should be anticipated in neonates with congenital syphilis. Preventive strategies such as intravenous fluids and early feeding should be instituted, together with regular dextrose monitoring during the first 24 hours after birth.

The majority $(68 \%)$ of neonates in the present study required maximal intervention and care. The cost implications of caring 


\begin{tabular}{|c|c|c|c|}
\hline Variable & Deaths $(N=19), n(\%)$ & Survivors $(N=31), n(\%)$ & $p$-value ${ }^{*}$ \\
\hline \multicolumn{4}{|l|}{ Gender } \\
\hline Male & $12(63)$ & $17(55)$ & 0.563 \\
\hline Female & $7(37)$ & $14(45)$ & 0.563 \\
\hline \multicolumn{4}{|l|}{ Mode of delivery } \\
\hline NVD & $9(47)$ & $22(71)$ & 0.095 \\
\hline CS & $10(53)$ & $9(29)$ & 0.095 \\
\hline \multicolumn{4}{|l|}{ Gestational age (weeks) } \\
\hline $25-29$ & $4(21)$ & $5(16)$ & 0.660 \\
\hline $30-34$ & $8(42)$ & $9(29)$ & 0.343 \\
\hline $35-37$ & $6(32)$ & $10(32)$ & 0.960 \\
\hline$\geq 38$ & $1(5)$ & $7(23)$ & 0.105 \\
\hline \multicolumn{4}{|l|}{ Birth weight (g) } \\
\hline $800-1000$ & $2(11)$ & 1(3) & 0.291 \\
\hline $1001-1500$ & $6(32)$ & $7(23)$ & 0.481 \\
\hline $1501-2000$ & $5(26)$ & $8(26)$ & 0.968 \\
\hline $2001-2500$ & $4(21)$ & $7(23)$ & 0.899 \\
\hline $2501-3000$ & $2(11)$ & $3(10)$ & 0.923 \\
\hline$>3000$ & 0 & $5(16)$ & 0.065 \\
\hline \multicolumn{4}{|l|}{ Need for resuscitation } \\
\hline Yes & $19(76)$ & $6(19)$ & 0.065 \\
\hline $\mathrm{CPAP} / \mathrm{basic}$ resuscitation & $6(32)$ & $4(13)$ & 0.109 \\
\hline Intubation and/or CPR & $11(58)$ & $2(6)$ & $<0.001$ \\
\hline Intubation and CPR and adrenaline & $2(11)$ & 0 & 0.140 \\
\hline \multicolumn{4}{|l|}{ Laboratory results } \\
\hline Hypoglycaemia & $12(63)$ & $15(48)$ & 0.309 \\
\hline Anaemia & $11(58)$ & $10(32)$ & 0.075 \\
\hline Coagulopathy & $10(53)$ & $7(23)$ & 0.029 \\
\hline Thrombocytopenia & $8(42)$ & $12(39)$ & 0.811 \\
\hline Long-bone changes & $4(21)$ & $7(23)$ & 0.899 \\
\hline \multicolumn{4}{|l|}{ Level of care } \\
\hline ICU admission & $18(95)$ & $16(52)$ & 0.002 \\
\hline Intubation and mechanical ventilation & $18(95)$ & $11(35)$ & $<0.001$ \\
\hline Need for inotropic support & $12(63)$ & $7(23)$ & 0.004 \\
\hline Need for blood products & $12(63)$ & $13(42)$ & 0.145 \\
\hline Blood transfusions & $11(58)$ & $11(35)$ & 0.121 \\
\hline Platelet transfusions & $6(32)$ & $8(26)$ & 0.659 \\
\hline Treatment of DIC & $12(63)$ & $14(45)$ & 0.216 \\
\hline
\end{tabular}

\section{Table 3. Abnormal CUS findings}

\begin{tabular}{|c|c|c|c|}
\hline Findings & Total number $(N=23), n$ & Survivors $(N=8), n$ & Deaths $(N=15), n$ \\
\hline Mild changes & 9 & 5 & 4 \\
\hline PV flares only & 1 & 1 & 0 \\
\hline Grade 1 or 2 IVHs & 8 & 4 & 4 \\
\hline Moderate/uncertain changes & 7 & 3 & 4 \\
\hline Grade 3 IVH (unilateral) & 2 & 2 & 0 \\
\hline 'Cerebral oedema’ & 5 & 1 & 4 \\
\hline Severe changes & 7 & 0 & 7 \\
\hline Bleeds with PVL & 1 & 0 & 1 \\
\hline Bleeds with cortical infarcts and PVL & 1 & 0 & 1 \\
\hline Bilateral grade 4 IVHs with hydrocephalus and PVL & 2 & 0 & 2 \\
\hline Bleeds with dilated ventricles & 3 & 0 & 3 \\
\hline
\end{tabular}


Table 4. Significant risk factors for death

\begin{tabular}{llll}
\hline & & \multicolumn{2}{c}{ Significance } \\
\cline { 3 - 4 } Risk factors for death & RR of death & $\mathbf{9 5 \%}$ CI & -value \\
\hline Hydrops fetalis & 2.6 & $1.4-4.8$ & 0.003 \\
Low 1-minute Apgar score $(<5)$ & 3.4 & $1.6-7.7$ & 0.001 \\
Low 5-minute Apgar score ( $\leq 5)$ & 2.9 & $1.5-5.3$ & 0.001 \\
Coagulopathy & 2.2 & $1.1-4.3$ & 0.030 \\
Abnormal cranial ultrasound scan (excludes PV flares and mild IVHs) & 3.5 & $1.8-6.9$ & $<0.001$ \\
PV = periventricular; IVH = intraventricular haemorrhage. & & &
\end{tabular}

for neonates with congenital syphilis are well known. In addition to preventing significant neonatal morbidity and mortality, this is the rationale behind screening for syphilis even in areas with a low prevalence of the disease. ${ }^{[32]}$ One study quoted adjusted costs of caring for an infant with syphilis that were more than three times that of caring for an infant without syphilis. ${ }^{[33]}$ Apart from the cost implications, prolonged hospital admissions have a psychological impact on the establishment of maternal-infant bonding.

Two strong predictors of mortality were low 1- and 5-minute Apgar scores. Other predictors were the need for early intubation or inotropes, and coagulopathy at birth. There was a statistically significant increase in mortality associated with severe CUS abnormalities. We could find no other studies with detailed descriptions of CUS findings in neonates with congenital syphilis, although the Baragwanath Hospital experience showed that IVH had a $74 \%$ positive predictive value for mortality. ${ }^{[10]}$ However, in our study there were probably confounding variables associated with these abnormalities, related to prematurity and birth asphyxia. Owing to our small sample size we did not correct for these confounders. Hydrops fetalis was a statistically significant independent risk factor for mortality, a similar finding to the Baragwanath Hospital experience with $100 \%$ mortality in the hydropic neonates. ${ }^{[10]}$

Disease notification is a vital public health initiative, and its use as a monitoring tool helps to evaluate the extent of the problem. In this study, notification of congenital syphilis was extremely poor, with just over half of the study population being notified. The identification of poor notification by health workers was demonstrated by Ballot and Rothberg ${ }^{[34]}$ in Johannesburg in the early 1990s, and yet more than 20 years later we are still failing in this regard.

\section{Study limitations}

The study has important limitations: it was retrospective and subject to data quality variation such as lack of booking data and partner tracing. The study referred to a specific tertiary neonatal population, so its conclusions are not generalisable to other populations.

\section{Conclusions}

Despite a large proportion of pregnant women having access to antenatal care and syphilis screening, over 50 infants with congenital syphilis were admitted to a tertiary unit in Cape Town over a 3-year period. The neonatal consequences of this disease warrant intensive care and intervention, with high mortality. This study adds to the existing body of research, particularly with regard to predictors of outcome in a tertiary neonatal setting. Further research may be required to explore some clinical findings such as abnormal CUSs and hypoglycaemia. Poor maternal diagnosis and treatment is a product of deficient health-seeking behaviour as well as modifiable health worker-related failures with poor adherence to public health initiatives such as disease notification and partner tracing. These factors are longstanding, highlighting the need to establish governance and audit processes and address the continuing socioeconomic and sociocultural barriers that mothers face as a way forward in ultimately eliminating this entirely preventable disease.

\section{Declaration. None.}

Acknowledgements. None.

Author contributions. All the authors contributed equally to this work.

Funding. None.

Conflicts of interest. None.

1. Gomez GB, Kamb ML, Newman LM, Mark J, Broutet N, Hawkes SJ. Untreated maternal syphilis and adverse outcomes of pregnancy: A systematic review and meta-analysis. Bull World Health Organ 2013;91:217-226. https://doi.org/10.2471/BLT.12.107623

2. Wijesooriya NS, Rochat RW, Kamb ML, et al. Global burden of maternal and congenital syphilis in 2008 and 2012: A health systems modelling study. Lancet Glob Health 2016;4(8):525-533. https://doi. org/10.1016/S2214-109X(16)30135-8

3. Peeling R, Mabey D, Fitzgerald D, Watson-Jones D. Avoiding HIV and dying of syphilis. Lancet 2004:364(9445):1561-1563. https://doi.org/10.1016/S0140-6736(04)17327-3

4. Walker DG, Walker GJA. Forgotten but not gone? The continuing scourge of congenital syphilis. Lancet Infect Dis 2002;2(7):432-436. https://doi.org/10.1016/S1473-3099(02)00319-5

Lancet Infect Dis 2002;2(7):432-436. https://doi.org/10.1016/S1473-3099(02)00319-5 5. Metrics GH. Global, regional, and national age-sex specific mortality for 264 causes of death, 1980
2016: A systematic analysis for the Global Burden of Disease Study 2016. Lancet 2017;390(10100):1151 2016: A systematic analysis for the Global Burden of Dise

6. Taylor M, Gliddon H, Nurse-Findlay S, et al. Revisiting strategies to eliminate mother-to-child transmission of syphilis. Lancet Glob Health 2018;6(1):26-28. https://doi.org/10.1016/S2214109X(17)30422-9

National Department of Health, South Africa. National Antenatal Sentinel HIV \& Syphilis Survey Report. 2015

8. Perinatal Problem Identification Programme. 2013. https://www.ppip.co.za/ (accessed 1 August 2019).

9. Hopkins KL, Doherty T, Gray G. Will the current National Strategic Plan enable South Africa to end AIDS, tuberculosis and sexually transmitted infections by 2022? South Afr J HIV Med 2018;19(1):a796. https://doi.org/10.4102/saihivmed.v19i1.796

10. Hauptfleisch CJ, Saloojee H, Cooper P. Should symptomatic congenital syphilitics be offered Hauptfleisch CJ, Saloojee H, Cooper P. Should symptomatic congenital syphilitics be offered
ventilation: The Baragwanath experience. Presented at the 15th Conference on Priorities in Perinatal ventilation: The Baragwanath experience. Presented at the 15 th Conference

11. Tooke L, Horn AR, Harrison MC. HIV transmission to extremely low birth weight infants. Pediatr Tooke L, Horn AR, Harrison MC. HIV transmission to extremely low birth
Infect Dis J 2013;32(1):36-38. https://doi.org/10.1097/INF.0b013e318270097e

12. Ballard JL, Khoury JC, Wedig K, Wang L, Eilers-Walsman BL, Lipp R. New Ballard Score, expanded to include extremely premature infants. J Pediatr 1991;119(3):417-423. https://doi.org/10.1016/S00223476(05)82056-6

13. Papile L-A, Burstein J, Burstein R, Koffler H. Incidence and evolution of subependymal and intraventricular hemorrhage: A study of infants with birth weights less than $1,500 \mathrm{gm}$. J Pediatr 1978;92(4):529-534. https://doi.org/10.1016/S0022-3476(78)80282-0

14. Cruz AR, Castrillón MA, Minotta AY, Rubiano LC, Castaño MC, Salazar JC. Gestational and congenital syphilis epidemic in the Colombian Pacific Coast. Sex Transm Dis 2013;40(10):813-818. https://doi.org/10.1097/OLQ.0000000000000020

15. Caddy SC, Lee BE, Sutherland K, et al. Pregnancy and neonatal outcomes of women with reactive syphilis serology in Alberta, 2002 to 2006. J Obstet Gynaecol Canada 2011;33(5):453-459. https://doi. syphilis serology in Alberta, 2002 to 200

16. Sangtawesin V, Lertsutthiwong W, Kanjanapattanakul W, Khorana M, Horpaopan S. Outcome of maternal syphilis at Rajavithi Hospital on offsprings. J Med Assoc Thai 2005;88(11):1519-1525.

17. Almeida KC, Lindolfo LC, Alcântara KC. Sí lis em gestantes atendidas em uma unidade de saúde pública de Anápolis, Goiás, Brasil. RBCA 2009;41(3):1814

18. Kaswa R, Rupesinghe GFD, Longo-Mbenza B. Exploring the pregnant women's perspective of late booking of antenatal care services at Mbekweni Health Centre in Eastern Cape, South Africa. Afr J Prim Health Care Fam Med 2018;10(1):1-9. https://doi.org/10.4102/phcfm.v10i1.1300

19. Saloojee H, Velaphi S, Goga Y, Afadapa N, Steen R, Lincetto O. The prevention and management of congenital syphilis: An overview and recommendations. Bull World Health Organ 2004;82(6):424-432.

20. Meyer Sauteur PM, Trück J, Bosshard PP, et al. Congenital syphilis in Switzerland: Gone, forgotten, on the return. Swiss Med Wkly 2012;141:3-8. https://doi.org/10.4414/smw.2012.13325

21. Simms I, Tookey P, Goh B, et al. The incidence of congenital syphilis in the United Kingdom: February 2010 to January 2015. BJOG 2017;124(1):72-77. https://doi.org/10.1111/1471-0528.13950

22. Reyes MP, Hunt N, Ostrea EM, George D. Maternal/congenital syphilis in a large tertiary-care urban hospital. Clin Infect Dis 1993;17(6):1041-1046. https://doi.org/10.1093/clinids/17.6.1041

23. World Health Organization, Special Programme for Research \& Training in Tropical Diseases. The use World Health Organization, Special Programme for Research \& Training in Tropical Diseases. The use
of rapid syphilis tests. 2006. https://apps.who.int/iris/bitstream/handle/10665/43590/TDR_SDI 06.1 eng.pdf? sequence $=1$ \&is Allowed $=y$ (accessed 1 August 2019).

24. Muricy CL, Pinto VL. Congenital and maternal syphilis in the capital of Brazil. Rev Soc Bras Med Trop 2015;48(2):216-219. https://doi.org/10.1590/0037-8682-0168-2014 
25. World Health Organization. Global guidance on criteria and processes for validation: Elimination of mother-to-child transmission (EMTCT) of HIV and syphilis. 2014. https://www.who.int/ of mother-to-child transmission (EMTCT) of HIV and syphilis. 2014.
reproductivehealth/congenital-syphilis/emtc-gvac/en/ (accessed 1 August 2019).

reproductivehealth/congenital-syphilis/emtc-gvac/en/ (accessed 1 August 2019).
26. Bloland P, Slutsker L, Steketee RW, Wirima JJ, Heymann DL, Breman JG. Rates and risk factors for mortality during the first two years of life in rural Malawi. Am J Trop Med Hyg 1996;55(1 Suppl):82-86. https://doi.org/10.4269/ajtmh.1996.55.82

27. Frank D, Duke T. Congenital syphilis at Goroka Base Hospital: Incidence, clinical features and risk factors for mortality. P N G Med J 2000;43(1-2):121-126. https://www.ncbi.nlm.nih.gov/ pubmed/11407606 (accessed 6 August 2019).

28. Singh AE, Romanowski B. Syphilis: Review with emphasis on clinical, epidemiologic, and some biologic features. Clin Microbiol Rev 1999;12(2):187-209. https://doi.org/10.1128/CMR.12.2.187

29. Brion LP, Manuli M, Rai B, Kresch MJ, Pavlov H, Glaser J. Long-bone radiographic abnormalities as a sign of active congenital syphilis in asymptomatic newborns. Pediatrics 1991;88 (5):1037-1040. https:// www.ncbi.nlm.nih.gov/pubmed/1945608
30. Pieper CH, van Gelderen WF, Smith J, Kirsten GF, Mohrcken S, Gie RP. Chest radiographs of neonates with respiratory failure caused by congenital syphilis. Pediatr Radiol 1995;25(3):198-200. https://doi.

1. Nolt D, Sad R, Ko Nolt D, Saad R, Kouatli A, Moritz ML, Menon RK, Michaels M. Survival with hypopititis
congenital syphilis. Pediatrics 2002;109(4):e63. https://doi.org/10.1542/peds.109.4.e63

32. Schmid G. Economic and programmatic aspects of congenital syphilis prevention. Bull World Health Organ 2004;82(6):402-409.

33. Bateman D, Phibbs CS, Joyce T, Heagarty MC. The hospital cost of congenital syphilis. J Pediat 1997;130(5):752-758. https://doi.org/10.1016/S0022-3476(97)80018-2

34. Ballot DE, Rothberg AD. Congenital syphilis as a notifiable disease. S Afr Med J 1993;83(10):721-723.

Accepted 18 March 2019 